\title{
Structural elucidation, Identification, quantization of process related impurity in Hydralazine Hydrochloride HR/AM- LC MS/MS, NMR and FTIR technique
}

\author{
Manohar V. Lokhande ${ }^{1,}$ Nitin G. Rathod ${ }^{2,3}$ Mukesh Kumar Gupta ${ }^{2,3}$ \\ ${ }^{1}$ Department of Chemistry, Sathaye College, Vileparle (E), Mumbai-400057, Maharashtra, India \\ ${ }^{2}$ Department of Chemistry, Shri Jagdish Prasad Jhabarmal Tibrewala University, Vidya Nagari, Jhunjhunu, \\ Chudela-333001, India \\ ${ }^{3}$ IPCA Laboratories Ltd., Chemical Research Division, Kandivli Industrial Estate, Kandivli (W), Mumbai- \\ 400102, Maharashtra India \\ Corresponding authors Email:manohar2210@gmail.com
}

\begin{abstract}
Major process related unknown impurity associated with the synthesis of Hydralazine hydrochloride bulk drug was detected by high performance liquid chromatography (HPLC) and was subjected to high resolution accurate liquid chromatography mass spectroscopy (HR/AM-LCMS) for identification. The proposed impurity was isolated from Hydralazine hydrochloride active pharmaceutical ingredient (API) by preparative chromatographic method and was injected on HPLC for comparison of retention time with that of the unknown process related impurity in Hydralazine hydrochloride. The molecular ion peak of preparatively isolated impurity and that of unknown process related impurity in Hydralazine hydrochloride were compared for confirmation. The postulated structure was unambiguously confirmed with the help of HR/AM- LC MS/MS, NMR and FTIR data proposed to be 1-(2-phthalazin-1-ylhydrazino)phthalazine (Hazh Dimer). This impurity of Hydralazine hydrochloride is not been previously reported. A rapid Acquity H-class gradient method with runtime of 15.0min was developed for Quantitation on Unisphere Cyno column and validated for parameters such as accuracy, precision, linearity and range, robustness. The LOD and LOQ of method were $0081 \%$ and $0.0246 \%$ respectively.
\end{abstract}

Keywords: Acquity UPLC H-class, FTIR, Hydralazine hydrochloride, , HPLC, , HR/AM-LCMS, NMR, , structural elucidation

\section{Introduction}

Hydralazine hydrochloride (1(2H)-phthalazinone:1-Hydrazinophthalazine hydrochloride) [1] (fig.1), is a direct acting vasodilator which reduces blood pressure and peripheral resistance. It is used in the management of hypertension, usually in doses below 100mg daily by mouth, since higher doses are associated with an increased incidence of lupus erythematosus. It has also been given intravenously in the treatment of hypersensitive crises [2]. Hydralazine is usually used in combination with other drugs. In recent years, it has been found to be especially useful when used with beta-adrenergic blocking agents and diuretics [3].

The analytical methods on impurity detection and identification are reported in some literatures and these literatures were screened for the presence of impurities in Hydralazine hydrochloride drug. Hydralazine hydrochloride is a Pharmacopoeia product [4-7], synthetically prepared (fig.2) and was injected on HPLC method from EP and USP, a late eluting unknown impurity peak at retention time 51.0min (fig.3) and 96.6min (fig.4) in chromatogram was observed during analysis respectively [4][5]. We have screened the literatures for impurities, which were produced form different synthetic process and found that this unknown impurity was not reported in any of the synthetic process related to Hydralazine hydrochloride active pharmaceutical ingredient (API) [8][9][10].

The impurity profile of the drug substance is critical for its safety assessment and manufacturing process. It is mandatory to identify and characterize the impurities in pharmaceutical product, if present above the accepted limit of $0.10 \%$ [11]. In this present article complete characterization of this unknown impurity was done using HR/AM-LCMS/MS, NMR, IR and rapid Acquity UPLC H-class instrument method is developed for Quantitation of this unknown impurity. However, so far there is no published report, describing the complete characterization and Quantitation of this unknown process related impurity in Hydralazine hydrochloride API.

During process development studies, impurities were detected in both crude and pure samples of Hydralazine hydrochloride using a newly developed gradient reversed phase Acquity UPLC H-class method developed for rapid analysis for Quantitation of process related unknown impurity. A comprehensive study was undertaken for the identification of this impurity using HR/AM-LCMS/MS followed by isolation and further 
characterization by NMR and FTIR technique. This article also describes the analytical method validation by using Acquity H-class for quantitative determination of this unknown impurity.

\section{Experimental}

2.1 Materials and reagents: Samples of Hydralazine hydrochloride API were obtained from Ipca Laboratories Ltd., HPLC grade acetonitrile, Methanol and Sulphuric acid were purchased from Merck India, Sodium lauryl Sulphate was purchased from Sigma Aldrich life science USA and tetra butyl ammonium bromide was purchased from Loba Chemie Mumbai India. HPLC grade water was taken from Millipore India. Dimethyl sulphoxide - $d 6$ (for NMR) were purchased from Aldrich Chemical Co., USA.

2.2 Liquid chromatography: Samples were analyzed on Acquity H-class (Waters, Milford, MA, USA) LC system equipped with Photo diode array (PDA) detector. A Cayno Unisphere column $(50 \mathrm{~mm}$ x $2.1 \mathrm{~mm}$ i.d. 2.5 $\mu \mathrm{m})$ was used for chromatographic separation. The mobile phase consisting of A: $1.4 \mathrm{gm}$ of sodium lauryl sulphate and $0.75 \mathrm{gm}$ of tetra butyl ammonium bromide was dissolved in $1000 \mathrm{~mL}$ of water and $\mathrm{pH}$ was adjusted with $0.10 \mathrm{~N}$ Sulphuric acid and mobile phase B: acetonitrile, with timed gradient programme $T_{\min } / \mathrm{A}: \mathrm{B}: T_{0} / 80: 20$; $T_{4} / 80: 20 ; T_{7} / 60: 40 ; T_{8} / 60: 40 ; T_{12} / 80: 20$ and $T_{15} / 80: 20$ with flow rate of $0.6 \mathrm{ml}$ per minute was used. The column oven temperature was maintained at $35^{\circ} \mathrm{C}$. The injection volume was $3.0 \mu \mathrm{L}$ and the detector wavelength was fixed at $230 \mathrm{~nm}$.

2.3 Liquid chromatography-high resolution accurate mass spectroscopy (LC-HR/AM-MS): The HR/AMLCMS/MS and MS/MS studies were performed on Q-Exactive Orbit trap mass spectrometer (Thermo Fisher Scientific Inc. Waltham, Massachusetts United States). HESI (Heated electron spray ionization) source was used for ionization. The spray voltage was maintained at $4.0 \mathrm{kV}$, Auxiliary gas flow rate was kept at 10 and capillary temperature at $320^{\circ} \mathrm{C}$. Nitrogen was used as both sheath and auxiliary gas. Heater temperature was kept at $350^{\circ} \mathrm{C}$ and S-lens RF level at 55 . The mass to charge ratio $(\mathrm{m} / \mathrm{z})$ was scanned across the range from 50 to 750 amu at a resolution of 70,000 with positive ion polarity. MS/MS studies were carried out by keeping normalized collision energy of (Higher-energy collision dissociation) HCD at 40eV and an isolation width of 6amu. The HPLC consisted of an Agilent-1100 series quaternary gradient pump with a degasser, an auto sampler and column oven. A C18 column (Kromasil C18 column $150 \mathrm{~mm}$ x $4.6 \mathrm{~mm}$ i.d. $5 \mu \mathrm{m}$ ) was used for separation. The mobile phase consisting of A: Water and B: acetonitrile, with timed gradient programme $T_{\min } / \mathrm{A}: \mathrm{B}: T_{0} / 50: 50$; $T_{10} / 50: 50 ; T_{15} / 5: 95 ; T_{25} / 5: 95 ; T_{30} / 50: 50 ; T_{35} / 50: 50$ with flow rate of $1.0 \mathrm{ml}$ per minute was used.

2.4 NMR spectroscopy: ${ }^{1} \mathrm{H},{ }^{13} \mathrm{C}$ NMR and DEPT measurement of the isolated impurities were performed on AVANCE 400 (Bruker, Fallanden, Switzerland) instrument. The ${ }^{1} \mathrm{H}$ and ${ }^{13} \mathrm{C}$ chemical shift values were reported on the $\delta$ scale ( $\mathrm{ppm}$ ) relative to DMSO.

2.5 FTIR spectroscopy: The FTIR spectrum of isolated impurity was recorded in the solid state as KBr powder dispersion using (Perkin-Elmer, Beaconsfield, UK) spectrum one FT-IR spectrometer.

2.6 Preparative liquid chromatography: Impurity was isolated from the crude sample using Waters Auto purification system consisting of 2525 binary gradient pump, a 2487UV detector and 2767sample manager (Waters, Milford MA, USA). A Kromasil C18 column $(150 \mathrm{~mm} \times 21 \mathrm{~mm}$ i.d., particle size $5 \mu \mathrm{m})$ was used for the separation. The mobile phase was consisted of a mixture of water and acetonitrile in the ratio of 50:50 and was pumped at flow rate $25 \mathrm{ml} / \mathrm{min}$. The detection was monitored at $230 \mathrm{~nm}$.

2.7 Preparation of solutions for validation of Acquity UPLC H-class method: A test preparation of 1000ppm of Hydralazine hydrochloride bulk drug sample was prepared using diluents (mobile phase A 80: mobile phase B 20). A stock solution of Hazh Dimer was prepared by dissolving $0.05 \mathrm{mg} / \mathrm{mL}$ (50ppm). From this stock solution a standard solution containing $0.001 \mathrm{mg} / \mathrm{mL}(1.0 \mathrm{ppm})$ was prepared. System suitability solution was prepared by mixing Hydralazine hydrochloride and Hazh Dimer to give concentration of 10ppm each. For LOD LOQ and linearity study concentrations ranging from $1.50 \mathrm{ppm}$ to $0.050 \mathrm{ppm}$ of nine levels were prepared. For precision study 1.0ppm of Hazh Dimer solution was used. For accuracy study LOQ to $120 \%$ Hazh Dimer was spiked in 1000ppm Hydralazine hydrochloride.

\section{Results And Discussion}

3.1 Detection of impurity by HPLC: During the analysis of Hydralazine hydrochloride API using Pharmacopeia chromatographic purity method / related substances method a late eluting impurity was found, when chromatographic run time was increased to, approximately five fold to principal peak retention time. This method was not suitable to quantify this late eluting impurity since the peak area, sensitivity of 1000ppm 
solution of Hydralazine hydrochloride containing unknown impurity was too low to quantitative and also the runtime was high.

Further detection and confirmation was done to trace the impurity by HPLC, by injecting the crude Hydralazine Hydrochloride to check if this impurity was coming from crude Hydralazine hydrochloride. It was observed that this unknown impurity is process related impurity and was present in crude sample and even after purification it remains in pure Hydralazine Hydrochloride API (fig. 3 and 4).

3.2 Identification of impurities by HR/AM-LCMS/MS: HR/AM-LCMS and MS/MS were performed as per the method described in the section 2.3 to generate the mass data for the impurity. The impurity of interest was eluted at retention time $6.0 \mathrm{~min}$ (fig. 5) which exhibits a protonated molecular ion peak $[\mathrm{M}+\mathrm{H}]^{+} 289$.

The impurity was isolated as described in section 2.6 and this impurity was analyzed by High resolution accurate mass spectrometry giving $[\mathrm{M}+\mathrm{H}]^{+} 289.11963$ and MS/MS of 272.09298 (fig.6). The theoretical atomic formula probability was shown by Xcalibur software for 289.11963 were $\mathrm{C}_{16} \mathrm{H}_{13} \mathrm{~N}_{6}$. PPM error was calculated using formula [12]:

Error in ppm for Molecular ion $=$ Theoretical value - Actual Value $\times 10^{6}$

$$
\begin{gathered}
\begin{array}{c}
\text { Theoretical Value } \\
=\frac{289.12017-289.11963}{289.12017} \times 10^{6} \\
=1.87 \mathrm{ppm}
\end{array}
\end{gathered}
$$

$1.87 \mathrm{ppm}$ error is highly acceptable for characterization of $[\mathrm{M}+\mathrm{H}]^{+}$of unknown structure using HR/AM- LCMS.

Error in ppm for MS/MS

$$
\begin{gathered}
=\frac{\text { Theoretical value }- \text { Actual Value }}{\text { Theoretical Value }} \times 10^{6} \\
=\frac{272.09307-272.09298}{272.09307} \times 10^{6} \\
=3.3 \mathrm{ppm}
\end{gathered}
$$

$3.3 \mathrm{ppm}$ error is highly acceptable for characterization of unknown structure using HR/AM- LCMS/MS. Though the mass error was low it was mandatory to confirm the structure by NMR. Hence NMR of impurity and the product was carried out for comparison and further confirmation of structure.

3.3 Brief synthetic preparation of Hydralazine Hydrochloride:Phthalazin-1(2H)-one when treated with $\mathrm{POCl}_{3}$ at $75^{\circ} \mathrm{C}-85^{\circ} \mathrm{C}$ in the presence of 2-ethoxy ethanol and toluene gives rise to 1-chlorophtalazine, this reaction takes place by intramolecular chlorination by Vilsmeir Hack reaction [13]. The solvent is distilled under vacuum and the product containing 1-chlorophtalazine is reacted with hydrazine hydrate and 2-ethoxy ethanol at $65^{\circ} \mathrm{C}$ to $70^{\circ} \mathrm{C}$ for $2 \mathrm{hrs}, 15 \% \mathrm{HCl}$ is added to give wet crude Hydralazine hydrochloride. This crude Hydralazine hydrochloride is purified with charcoal, EDTA and methanol to give Hydralazine hydrochloride pure API refer (fig 2.).

3.4 Isolation and structural confirmation of unknown impurity by NMR and FTIR : During the synthesis of Hydralazine hydrochloride the unknown impurity formed was isolated using preparative chromatography described in section 2.6. The chromatographic purity was checked and found to be $99 \%$ (fig.7). ${ }^{1} \mathrm{H}$ and ${ }^{13} \mathrm{C}$ NMR spectral data (refer table no.1) confirmed the proposed structure. The MS/MS spectrum obtained for isolated compound of impurity using direct infusion mode was exactly same as MS/MS spectrum of 1-(2phthalazin-1-ylhydrazino) phthalazine the plausible mass fragmentation is given in (fig.8). The infrared spectrum of Hazh Dimer was scanned from 400 to $4000 \mathrm{~cm}^{-1}$ some of the absorption bands assigned are 3332 $\mathrm{cm}^{-1} 1$ for $\mathrm{N}-\mathrm{H}$ stretch secondary amine, 1590 for $\mathrm{C}=\mathrm{C}$ stretch and $\mathrm{CH}-\mathrm{N}$ for aromatic tertiary amine (fig.9).

3.5 Analytical Method Validation by HPLC: The validation study allowed the evaluation of the method for its suitability for regular analysis. The newly developed method for Hydralazine hydrochloride and Hazh Dimer impurity was validated according to ICH guidelines [14]. A typical chromatogram showing Hydralazine Hydrochloride and Hazh Dimer impurity (fig. 10)

3.5.1 Specificity: Specificity is the ability of analytical method to measure the analyte response in the presence of its potential impurities and degradants. The specificity of the Acquity UPLC H-class liquid method was determined by injecting individual impurity samples, wherein no interference was observed for any of the components.

The chromatograms were checked for the appearance of any extra peak. Peak purity of these was verified using a PDA detector. The peak purity of the principle and other chromatographic peaks was found to be satisfactory (fig. 11). 
3.5.2 Precision: The precision of the method was examined using six replicate injections of a standard solution. The relative standard deviation (RSD) was calculated for response (area) of Hazh Dimer impurity. The RSD for Hazh Dimer impurity was found to be $2.37 \%$ (refer Table no.2). The method precision was established by analyzing using six different preparations. The calculated RSD of these results was found to be within acceptable limit (fig.12).

3.5.3 Accuracy: The accuracy of the method was determined for the related substances by spiking of known amounts of a Hazh Dimer impurity in Hydralazine hydrochloride at levels, LOQ, $80 \%, 100 \%$ and $120 \%$ of the specified limit. The recoveries of impurities were calculated and are given in Table no. 3 (fig.13).

3.6 Limit of detection and limit of quantification: Detection limit (DL) and quantization limit (QL) was estimated as per ICH Q2 (R1). The limit of detection established for Hazh Dimer impurity was found to be $0.0081 \%$ and limit of quantification was found to be $0.0246 \%$.

3.6.1 Linearity: Linear calibration curve were obtained over the calibration range i.e. LOQ, 50\%, 80\%, 100\%, $120 \%$ and $150 \%$ at six concentration levels in triplicate. The results showed excellent correlation between the peak area and concentration of Hazh Dimer impurity of about 0.9989 Table no. 4 (fig. 14).

3.6.2 Robustness: In all the deliberately varied chromatographic conditions (column temperature and flow rate), no significant changes in results were observed

3.6.3 Solution stability: The solution stability of Hydralazine sample and Hazh Dimer impurity was carried out by keeping both solutions in tightly capped HPLC vials at $25^{\circ} \mathrm{C}$ for $8 \mathrm{hrs}$ in an Acquity UPLC H-class auto sampler no significant changes were observed in the peak area.

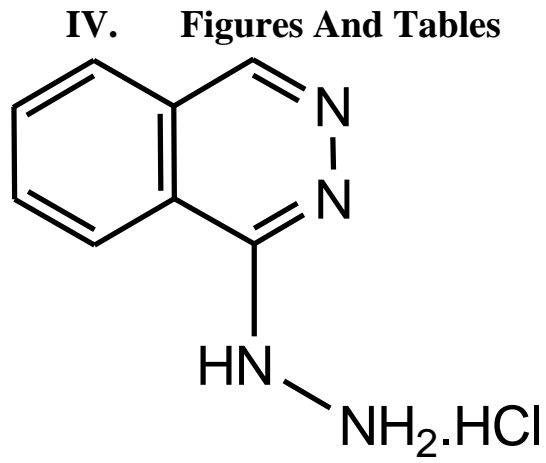

Fig.1 Structure of Hydralazine Hydrochloride<smiles>O=c1[nH]ncc2ccccc12</smiles>

Phthalazin-1(2H)-one

\section{Toluene 2. $\mathrm{POCl}_{3}, 75-80^{\circ} \mathrm{C}$ 3. 2-Ethoxy ethanol}<smiles>Clc1nncc2ccccc12</smiles>

1-chlorophthalazine

$$
\begin{aligned}
& \text { 1. } \mathrm{NH}_{2} \mathrm{NH}_{2} \cdot \mathrm{H}_{2} \mathrm{O}+2 \text {-Ethoxy ethanol } \\
& \text { 2. Heated } 2 \mathrm{hr} \text { at } 65-70^{\circ} \mathrm{C} \\
& \text { 3.pH } 3-4.5 \text { by } 15 \% \mathrm{HCl}
\end{aligned}
$$<smiles>NNc1nncc2ccccc12</smiles>

1. D. $\mathrm{M} \mathrm{H}_{2} \mathrm{O}$

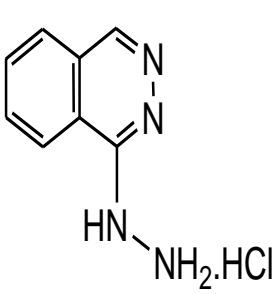


Fig.2 Synthetic Scheme for Hydralazine Hydrochloride

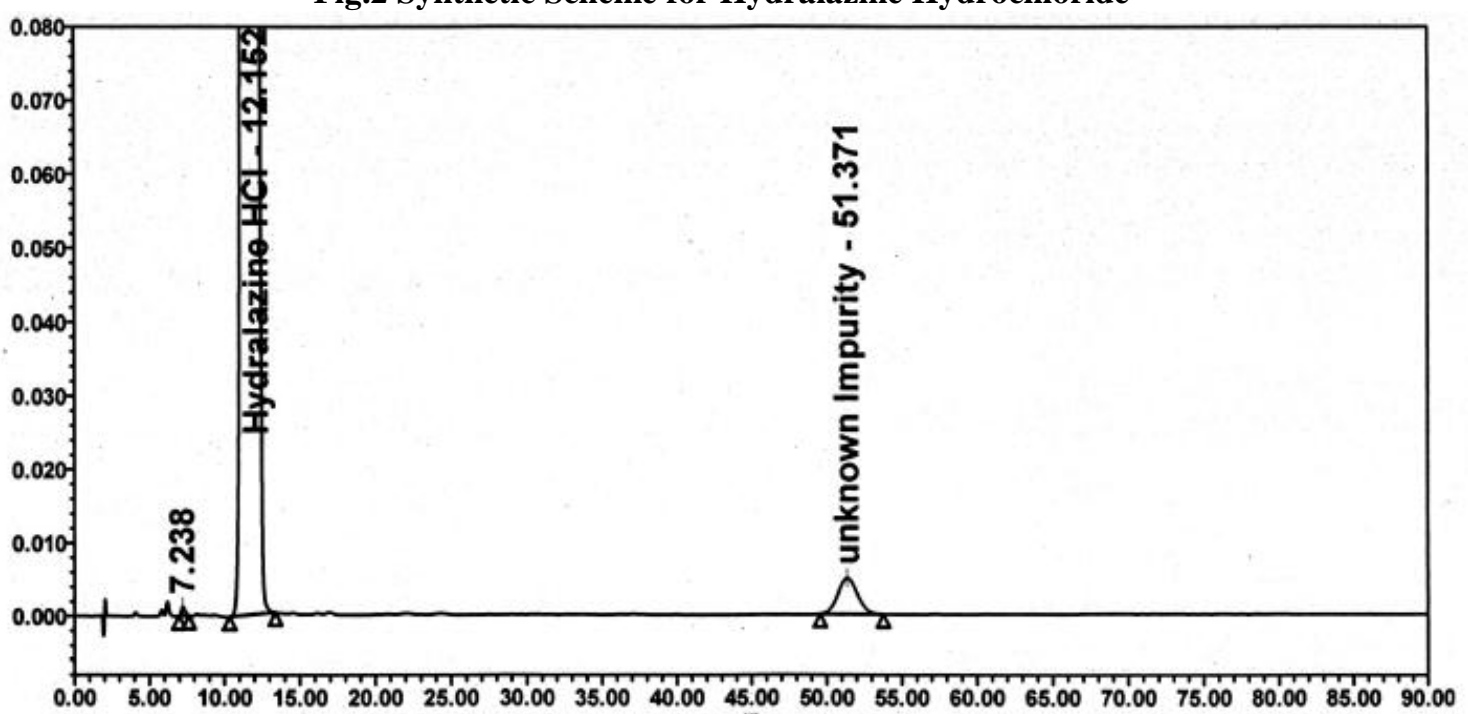

Fig. 3 Typical chromatogram of Hydralazine Hydrochloride EP Method showing unknown at 51.37min

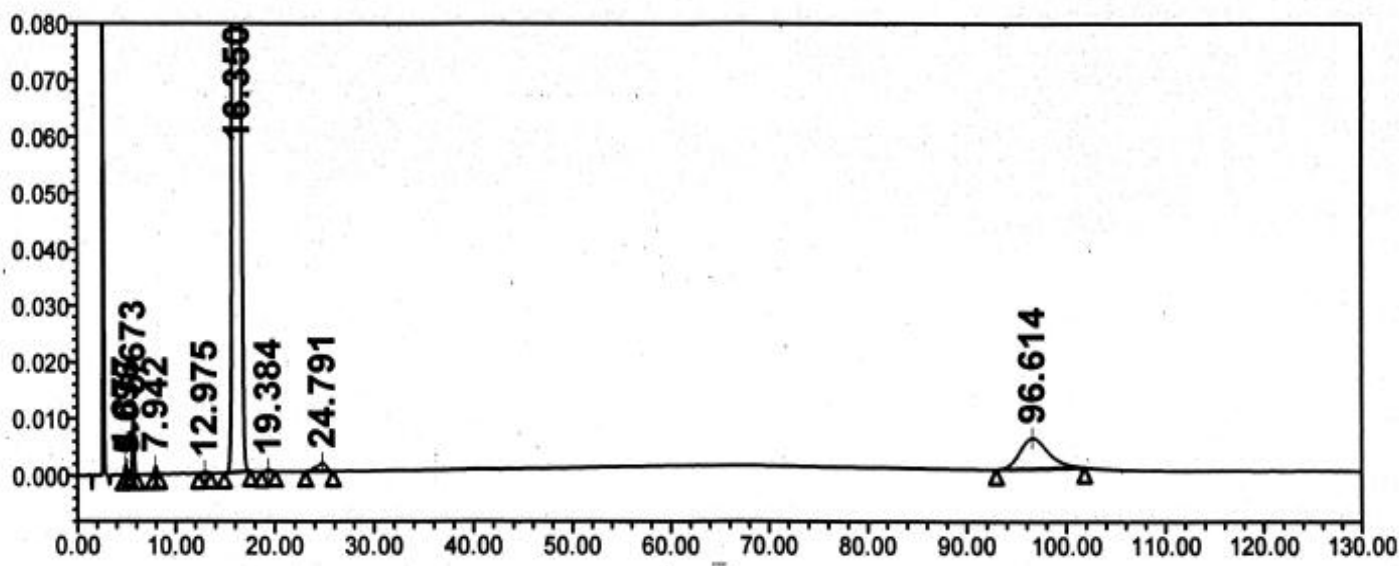

Fig.4 A typical chromatogram of Hydralazine Hydrochloride USP Method showing unknown at 96.61min RT: $0.00-30.00$ SM: $11 \mathrm{G}$

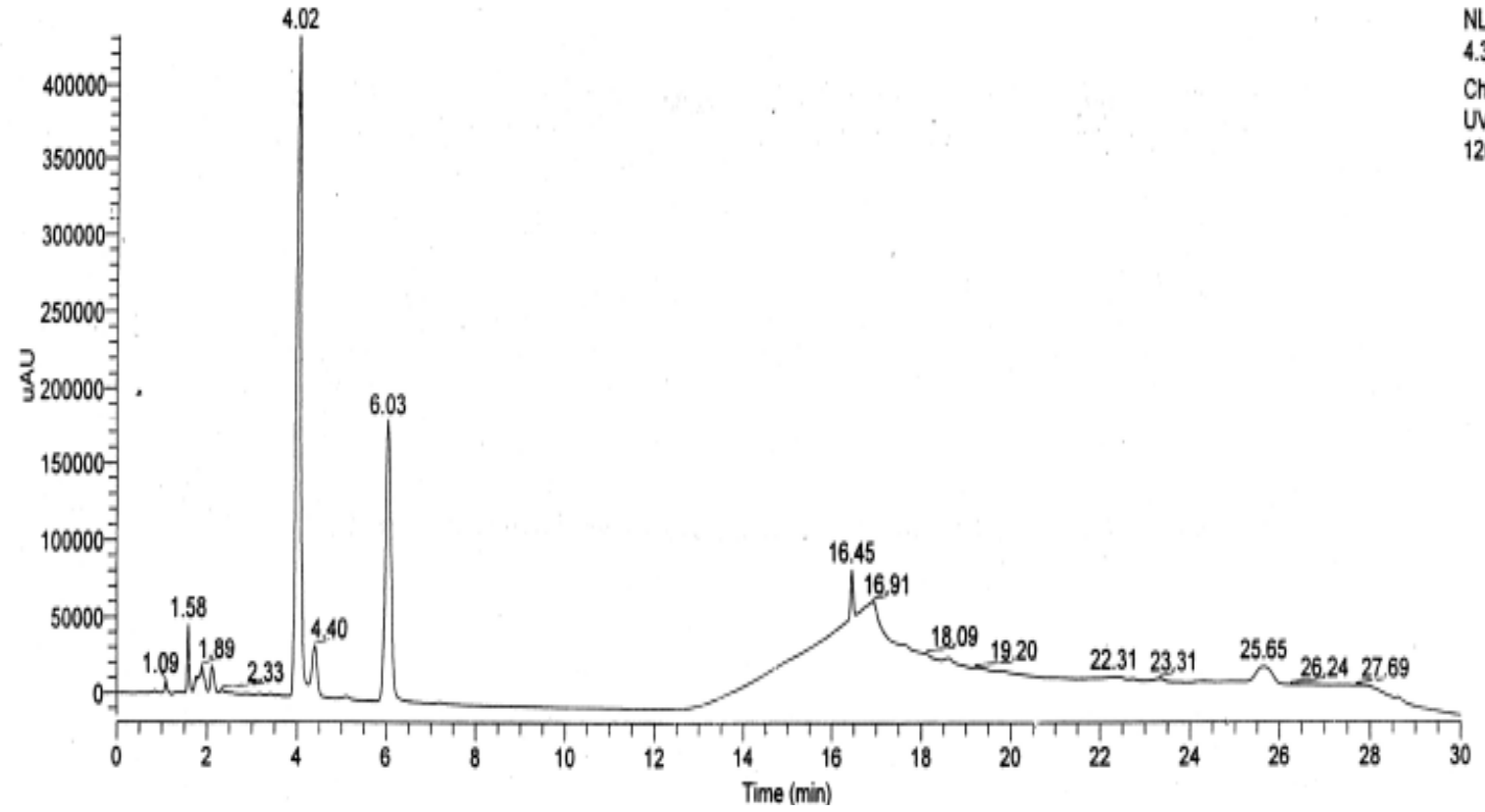

Fig. 5 A typical TIC chromatogram of unknown impurity at 6.03 minute 


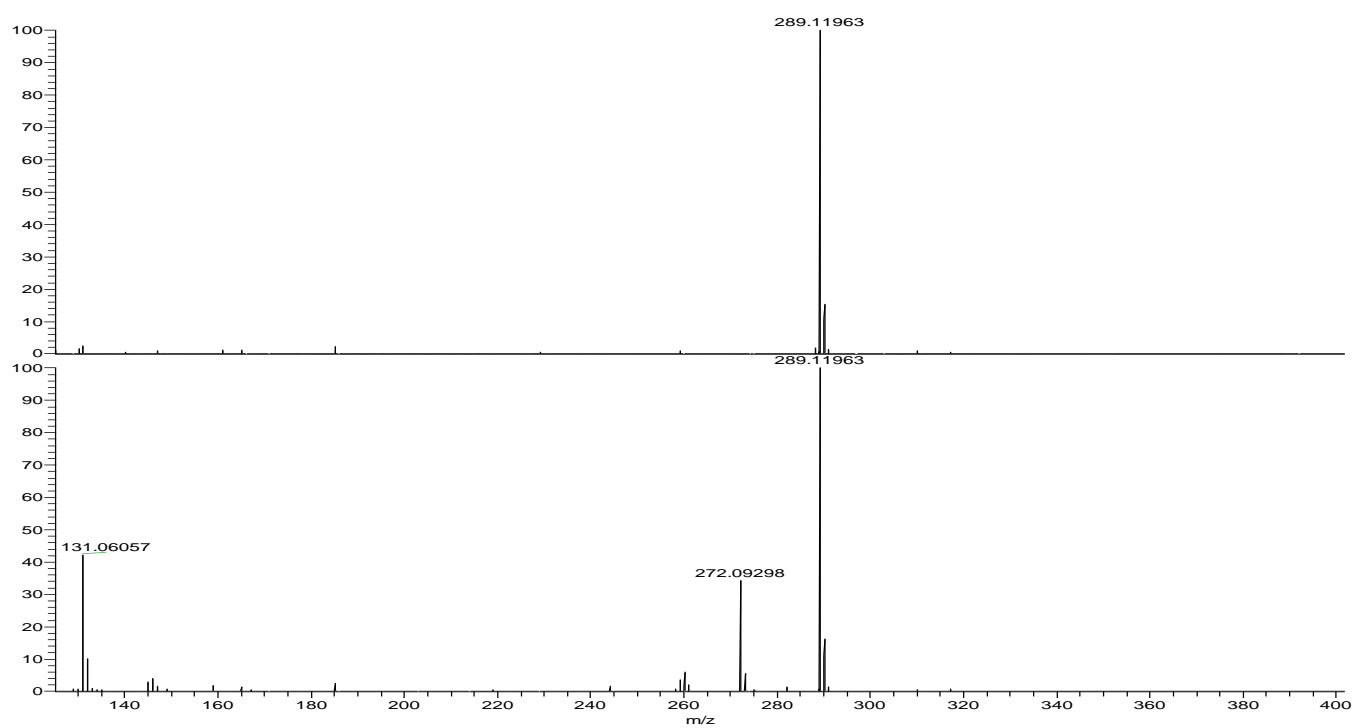

29051208\#43 RT: 0.29 AV O.33: 1.45 T: FT-OMS + P' ESIFul

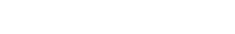

Fig.6 HR/AM- LC MS/ and MS/MS of Hazh Dimer impurity
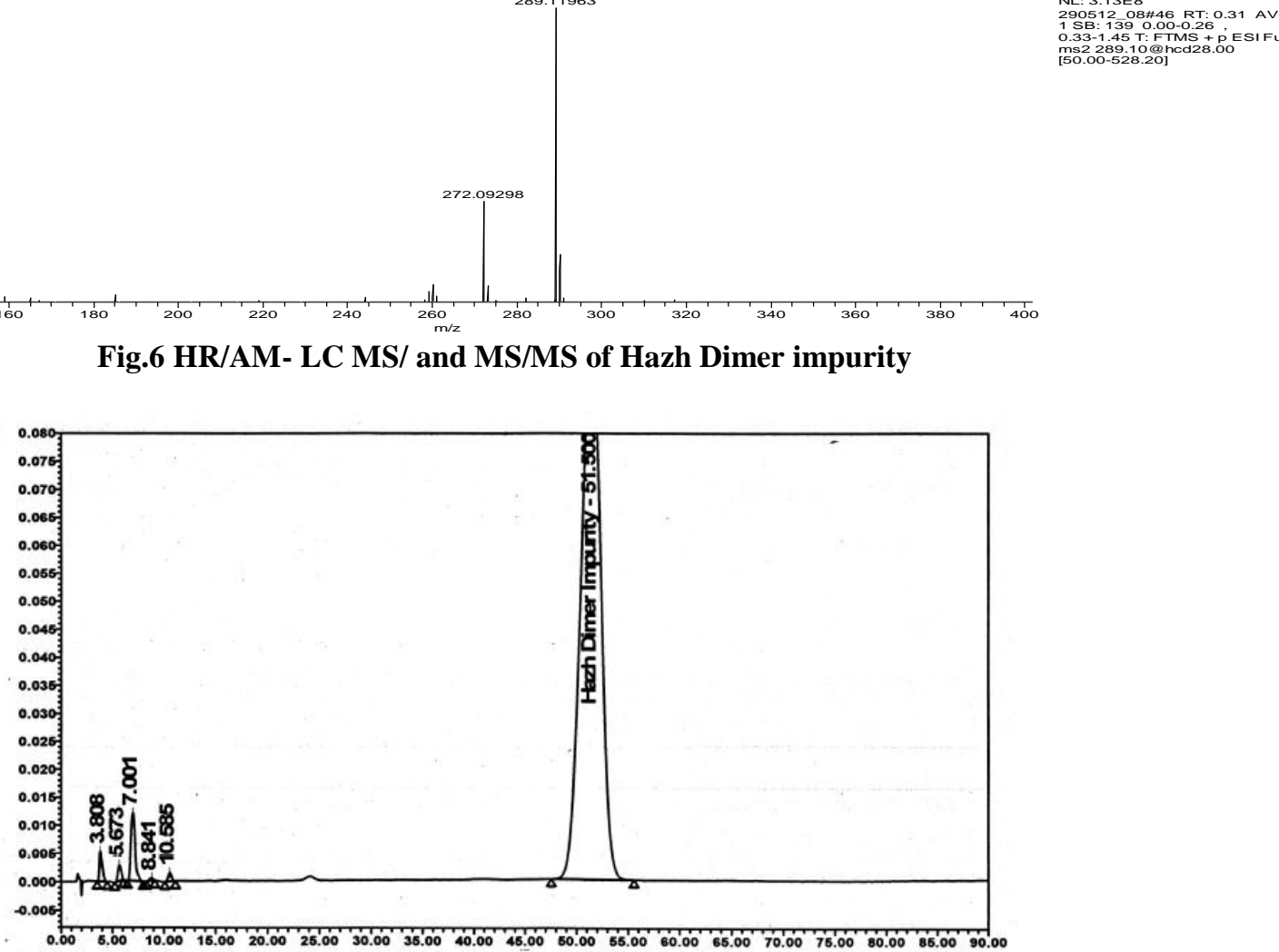
.33-1.45 T:FTMS + P'ESIFU

Fig.7 A typical chromatogram of preparative isolated Hazh Dimer impurity<smiles>c1ccc2c(NNc3nncc4cnnc(NNc5nncc6ccccc56)c34)nncc2c1</smiles>

$$
\mathrm{C}_{16} \mathrm{H}_{12} \mathrm{~N}_{6}
$$

Exact Mass: 288.11234

$\mathrm{C}_{16} \mathrm{H}_{13} \mathrm{~N}_{6}^{+}$

Exact Mass: 289.11962

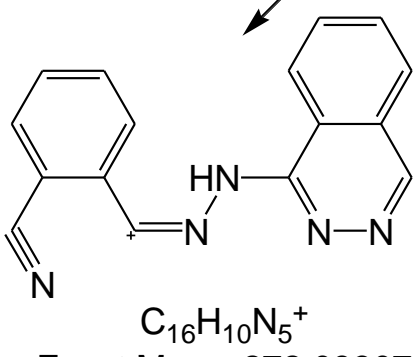

Exact Mass: 272.09307 
Fig.8 Plausible fragmentation for Hazh Dimer impurity

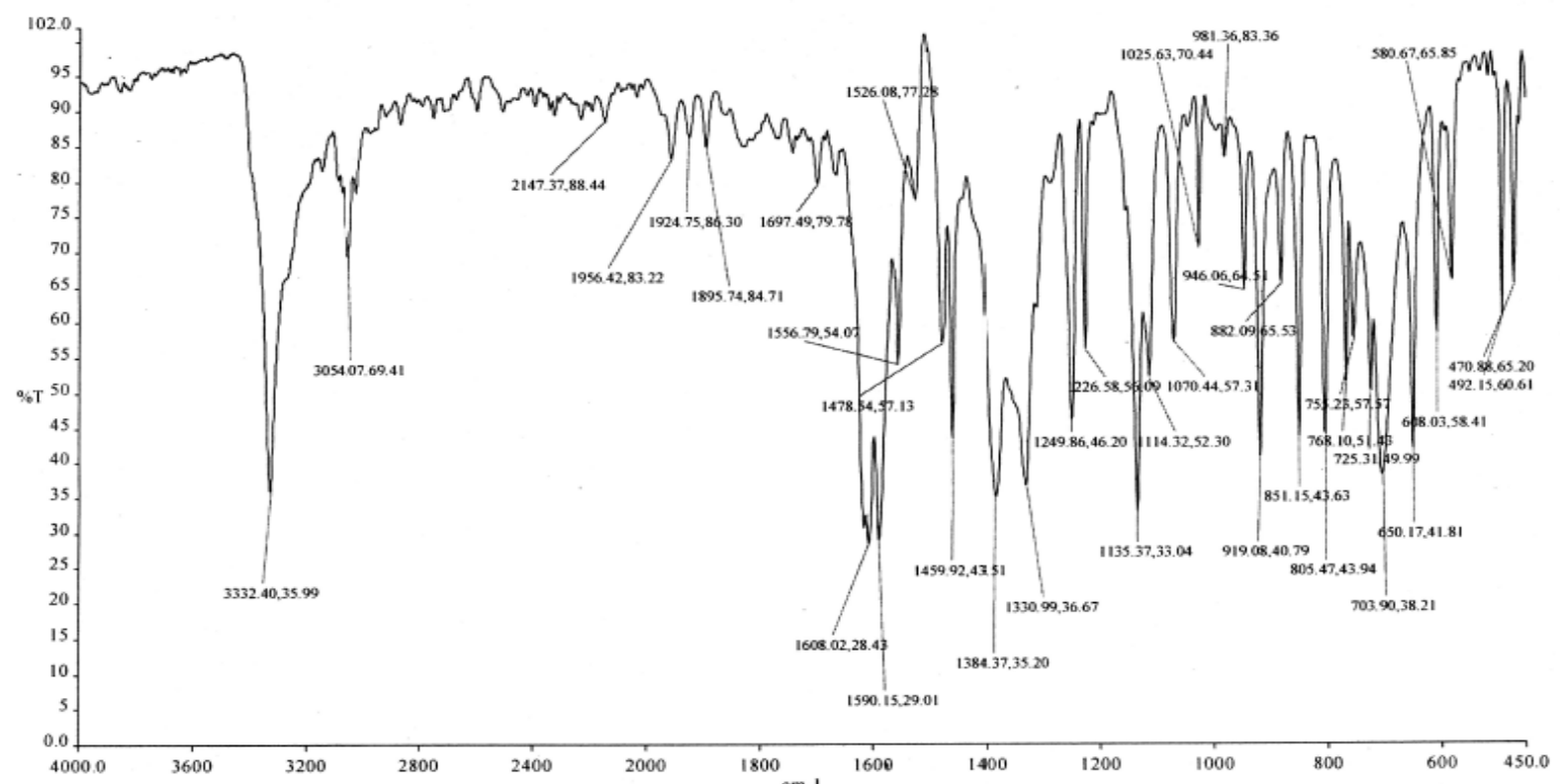

Fig.9 A FTIR spectra of Hazh Dimer impurity
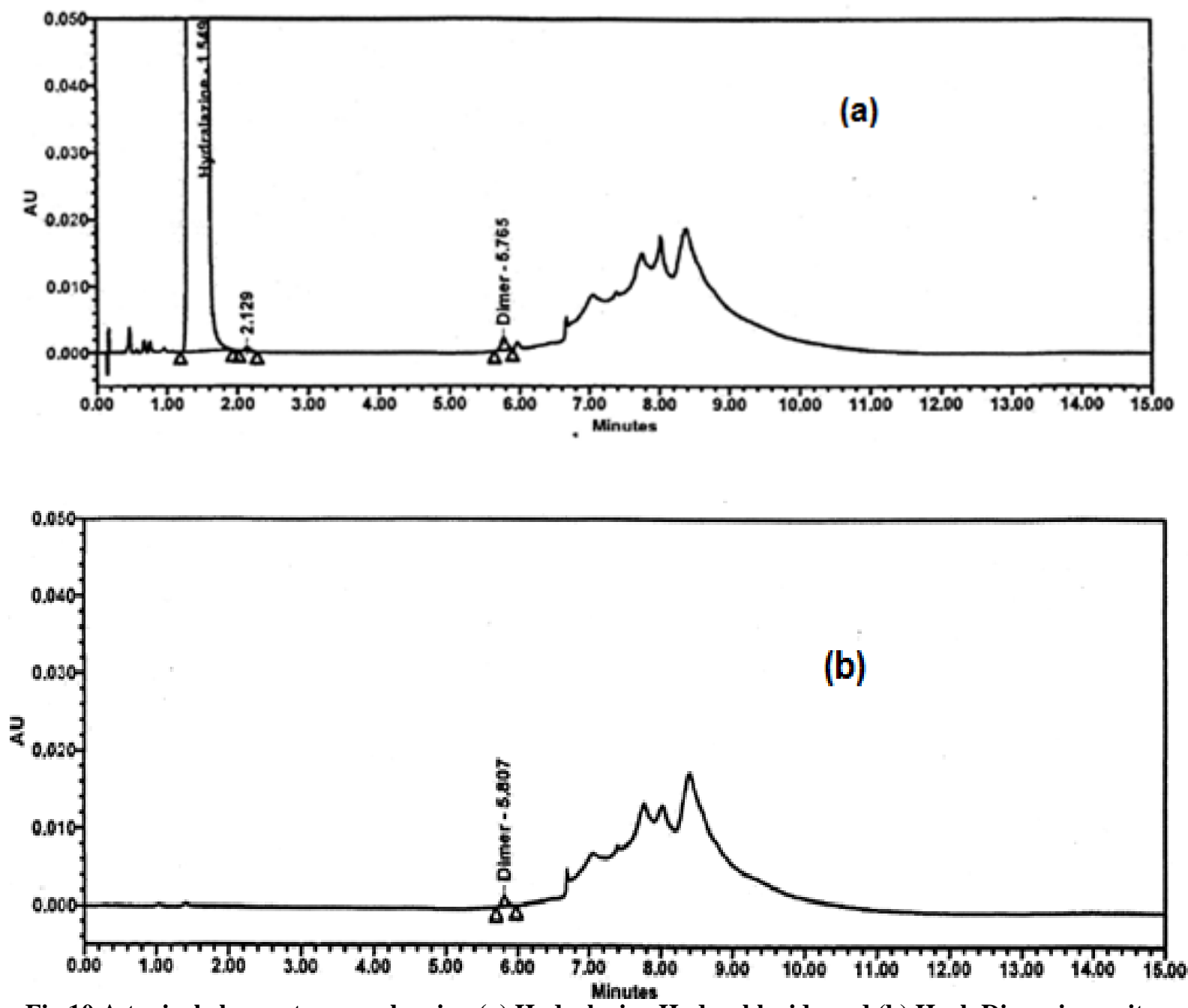

Fig 10 A typical chromatogram showing (a) Hydralazine Hydrochloride and (b) Hazh Dimer impurity using Acquity UPLC H-class 


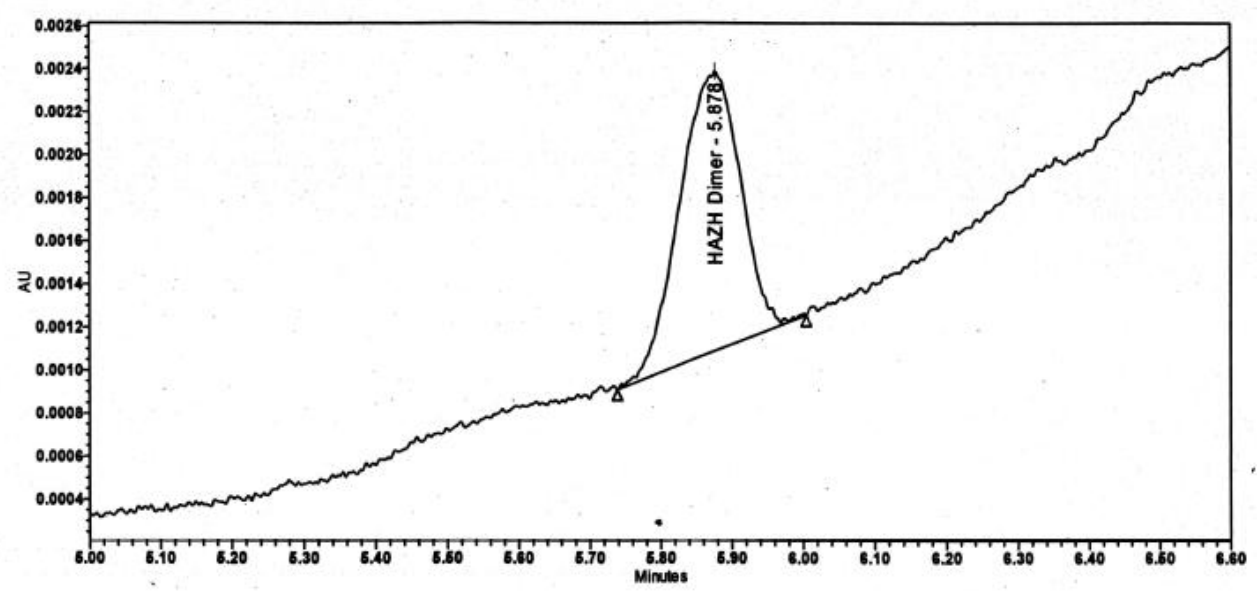

\begin{tabular}{|c|c|r|r|r|r|}
\hline & Name & Retention Time & $\begin{array}{c}\text { Purity1 } \\
\text { Angle }\end{array}$ & $\begin{array}{c}\text { Purity1 } \\
\text { Threshold }\end{array}$ & Area \\
\hline 1 & HAZH Dimer & 5.878 & 4.880 & 6.045 & 7739 \\
\hline
\end{tabular}

Fig.11 A typical chromatogram showing Peak purity (purity angle less than purity threshold) of Hazh Dimer

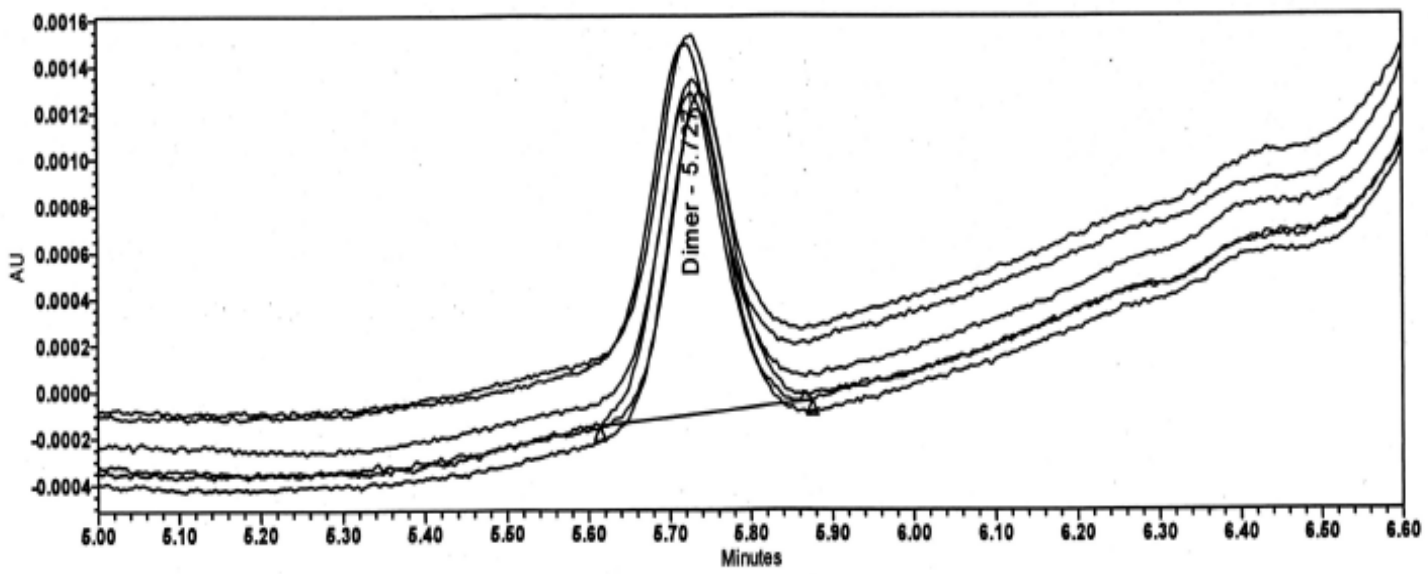

Fig.12 A typical chromatogram showing RSD for six replicate injections of Hazh Dimer

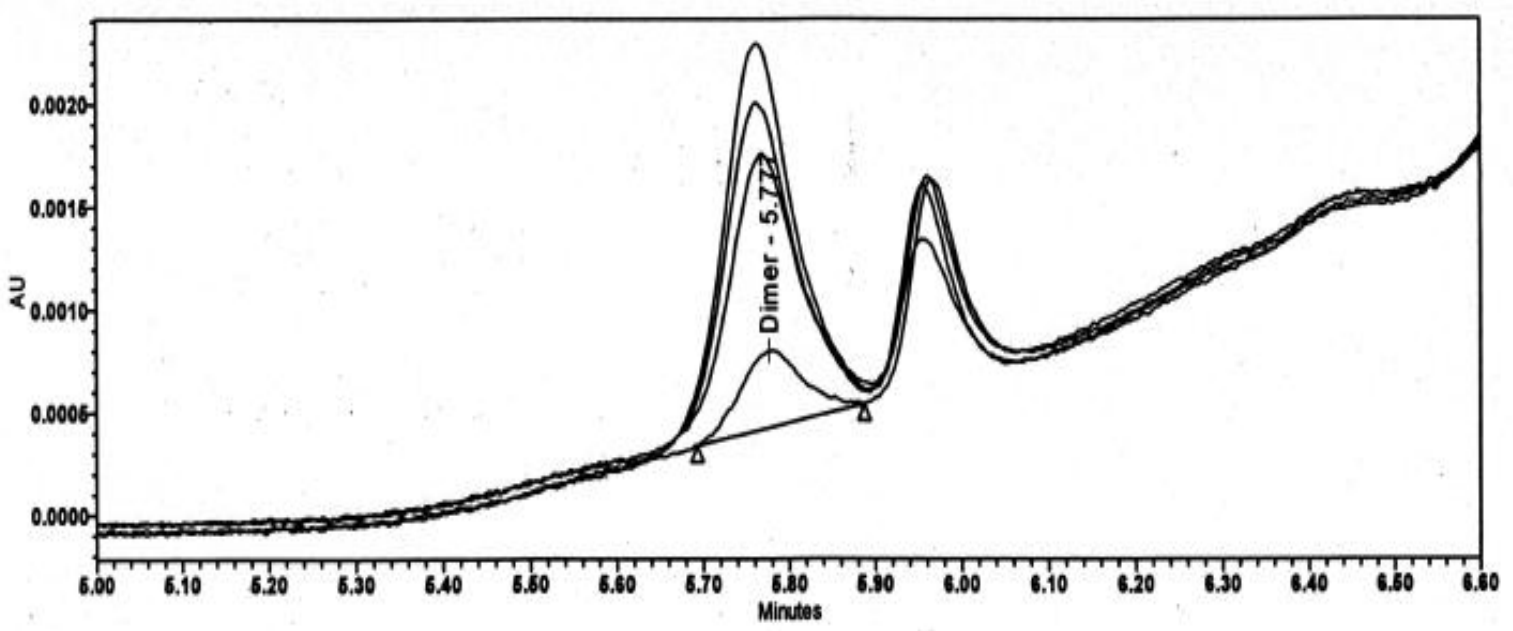

Fig.13 A overlay chromatogram showing spike of Hazh Dimer at LOQ, $80 \%, 100 \%, 120 \%$ in Hydralazine Hydrochloride 


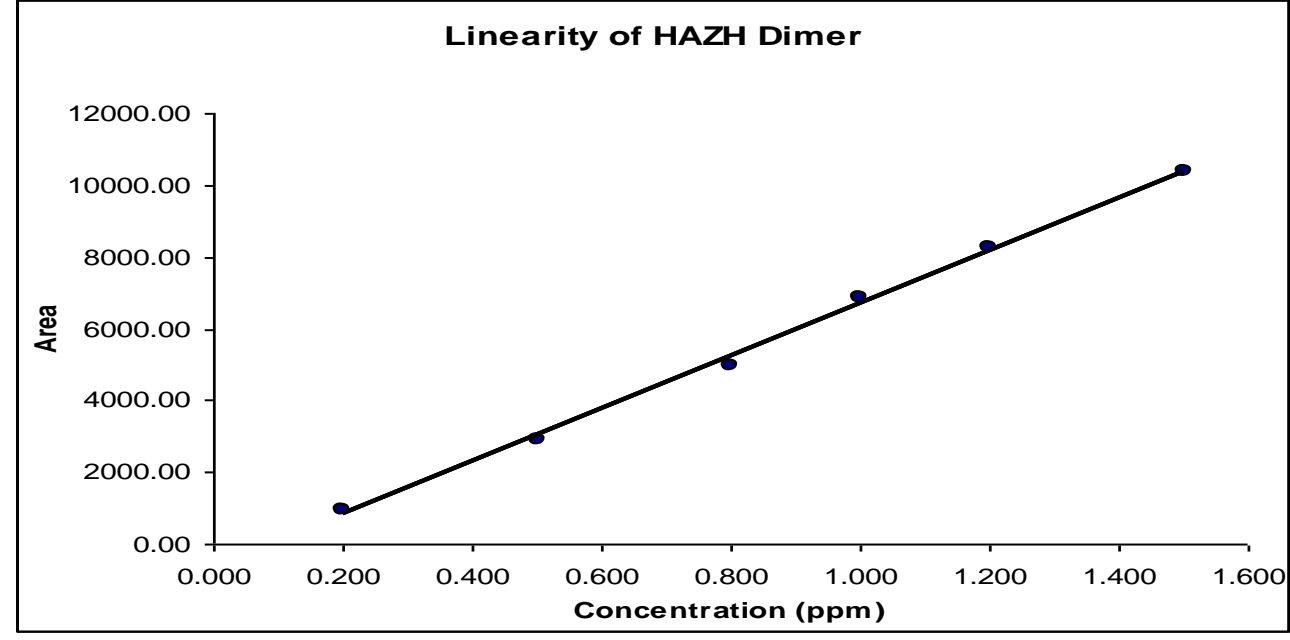

Fig.14 Linearity of Hazh Dimer

Table no.1: ${ }^{1} \mathrm{H}$ NMR and C-13 assignment for Hazh Dimer<smiles>c1ccc2c(NNc3nncc4ccccc34)nncc2c1</smiles>

\begin{tabular}{|c|c|c|c|c|}
\hline Position ${ }^{\mathrm{a}}$ & Integration & $\delta(\mathrm{ppm})$ & Multiplicity, $J(\mathrm{~Hz})^{\mathrm{a}}$ & $13 \mathrm{C} \delta(\mathrm{ppm})$ \\
\hline 1 & - & - & - & 144.3 \\
\hline 2 & - & - & - & - \\
\hline 3 & - & - & - & - \\
\hline 4 & $2 \mathbf{H}$ & 7.86 & $\mathbf{s}$ & 136.9 \\
\hline 5 & $2 \mathrm{H}$ & 7.57-7.66 & $\mathbf{m}$ & 127.1 \\
\hline 6 & $2 \mathrm{H}$ & $7.57-7.66$ & $\mathbf{m}$ & 131.7 \\
\hline 7 & $2 \mathbf{H}$ & $7.57-7.66$ & $\mathbf{m}$ & 131.6 \\
\hline 8 & $2 \mathrm{H}$ & $8.64-8.66$ & $\mathbf{m}$ & 126.3 \\
\hline 9 & - & - & - & 124.8 \\
\hline 10 & - & - & - & 128.1 \\
\hline 11 & $2 \mathbf{H}$ & 11.59 & $\mathbf{s}$ & - \\
\hline
\end{tabular}

${ }^{a}$ Refer the structural formula in Figure. ${ }^{\mathrm{b}}{ }^{\mathrm{l}} \mathrm{H}-{ }^{\mathrm{l}} \mathrm{H}$ coupling constants.

Table no. 2 Precision Study (Hazh Dimer Precision study)

\begin{tabular}{|c|c|c|c|c|}
\hline No of Injection & Retention time (min) & Area & Tailing factor & Theoretical Plates \\
\hline 1 & 5.727 & 7884 & 1.04 & 22363 \\
\hline 2 & 5.722 & 7741 & 1.01 & 23803 \\
\hline 3 & 5.733 & 7593 & 1.08 & 24885 \\
\hline 4 & 5.738 & 7604 & 1.04 & 25233 \\
\hline 5 & 5.728 & 7678 & 0.99 & 21613 \\
\hline 6 & 5.729 & 7341 & 1.07 & 25102 \\
\hline Mean & 5.730 & 181.1556 & 0.0343 & 23833 \\
\hline Std Dev & 0.0055 & $\mathbf{2 . 3 7}$ & $\mathbf{3 . 3 0}$ & 1534.0968 \\
\hline RSD & $\mathbf{0 . 1 0}$ & 7593 & 0.99 & $\mathbf{6 . 4 4}$ \\
\hline Min & 5.722 & & & 21613 \\
\hline Max & 5.738 & 7884 & & \\
\hline
\end{tabular}


Structural elucidation, Identification, quantization of process related impurity in Hydralazine

Table no. 3 Accuracy for Hazh Dimer

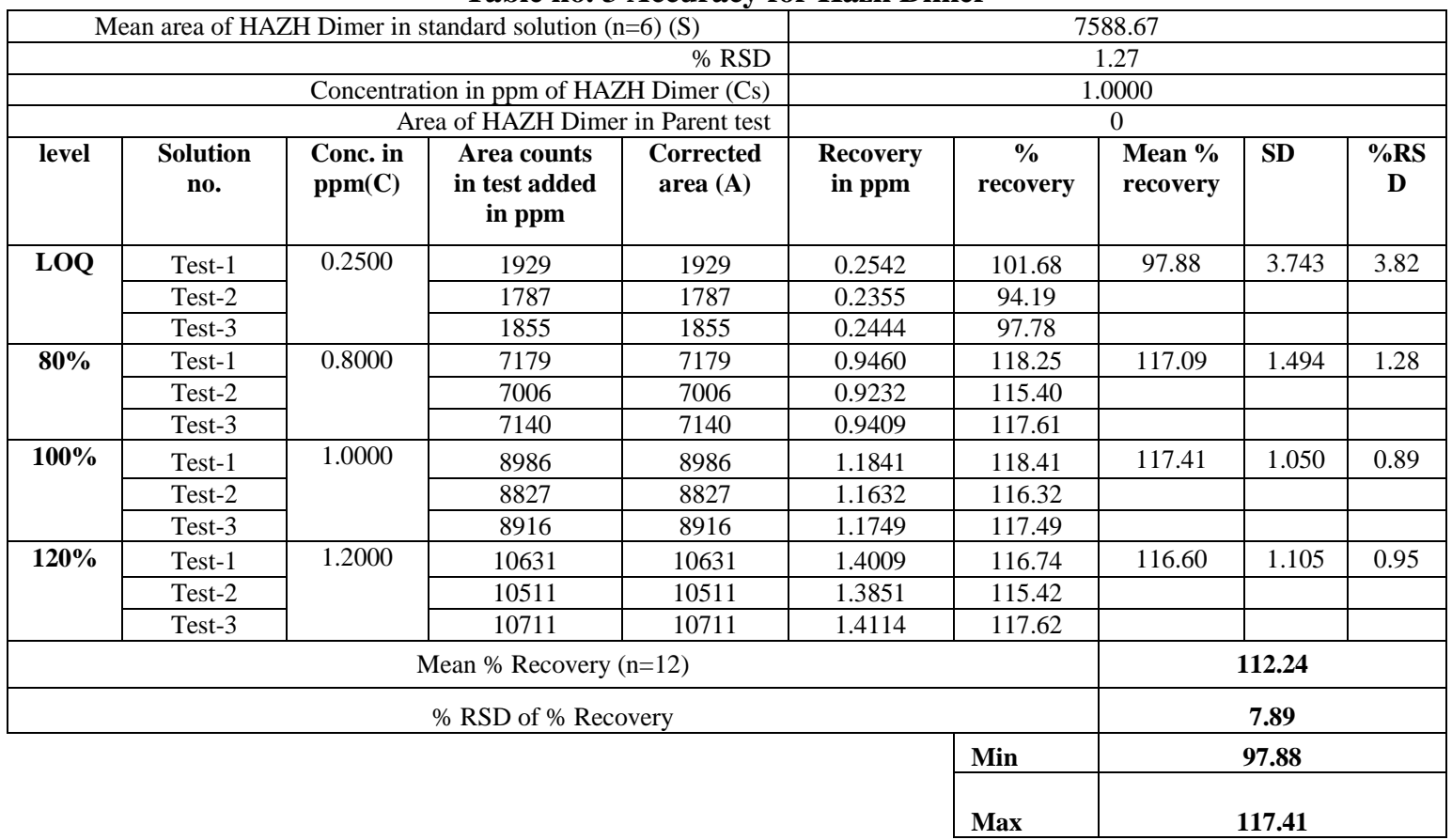

Table no. 4 Linearity of Hazh Dimer Linearity of HAZH Dimer

\begin{tabular}{|c|c|c|c|}
\hline Level & Conc.(ppm) & Area & Regression area \\
\hline Near LOQ Level & 0.200 & 975.33 & 820 \\
\hline $50 \%$ & 0.500 & 2909.33 & 3032 \\
\hline $80 \%$ & 0.800 & 4994.00 & 5244 \\
\hline $100 \%$ & 1.000 & 6864.67 & 6718 \\
\hline $120 \%$ & 1.200 & 8266.33 & 8193 \\
\hline $150 \%$ & 1.500 & 10402.00 & 10405 \\
\hline
\end{tabular}

\begin{tabular}{|c|c|}
\hline Correlation coefficient & 0.9989 \\
\hline Slope & 7373.14 \\
\hline Intercept & -654.77 \\
\hline
\end{tabular}

\section{Conclusion}

A major process related unknown impurity of Hydralazine hydrochloride was Isolation by using semipreparative method. The structural characterization of the isolated impurity was carried out by using HR/AMLC MS/MS and other modern spectroscopic (NMR and FTIR) techniques. The combined result of HR/AM- LC MS/MS, NMR and FTIR confirmed the structure of unknown impurity as1-(2-phthalazin-1-ylhydrazino) phthalazine. A rapid Acquity UPLC H-class liquid chromatographic method developed was successfully validated for control of 1-(2-phthalazin-1-ylhydrazino) phthalazine in Hydralazine Hydrochloride API.

\section{Acknowledgement}

The authors wish to thank, the manager, IPCA Laboratories limited, for providing necessary facilities for research work. Special Thanks to Dharmendra Singh, Kishor More, Usha Bhosle, Prathamesh Deshpandey, Lalita, Mayuri, Vishant for their cooperation and help. 


\section{References}

[1]. Merck Index, an encyclopedia of chemicals, drugs and biological, Fourteenth edition, Whitehouse Station,( Merck \& Co., New Jersey USA,2006) 824.

[2]. James E F Reynold, Kathleen Barfitt Martindale, the extra pharmacopoeia $31^{\text {st }}$ edition.( Published by the council of royal pharmaceutical society of Great Britain. , 1996 ) 885

[3]. Analytical profiles of drug substances Volume 8 Klaus Florey publication by Academic press. 283-314.

[4]. European pharmacopoeia. 2011, 2: 2191.

[5]. United States pharmacopoeia 36 National formulary Volume 2 pp 3818.

[6]. The Japanese pharmacopoeia sixteenth edition published by pharmaceutical and medical device regulatory science society of Japan. (Shibuya, Tokyo 150-0002 Japan,2012) 924

[7]. Pharmacopoeia of the people's republic of China, volume II. Chinese pharmacopoeia commission. Published by China medical science press (A-22, Northern Wenhuiyan road, Haidian district Bejing China, 2010) 100082.

[8]. United States patent application publication number US2007/0129546A1, 2007.

[9]. United States patent publication number US7531653B2, 2009.

[10]. United States patent publication number US 2009187018 A1 , 2009.

[11]. ICH International conference on harmonization, Impurities in New Drug Substances Q3A (R2),2006.

[12]. A. Gareth Brenton and A. Ruth Godfrey Accurate Mass Measurement: Terminology and Treatment of Data.,Institute of Mass Spectrometry, Grove Building, School of Medicine, Swansea University, Swansea, Wales, )United Kingdom Published by Elsevier Inc. on behalf of American Society for Mass Spectrometry,2010)

[13]. Bradford P Mundy, Michael G Ellerd and Frank G Favaloro Jr., Name reaction and reagents in organic synthesis.( A Jhon Wiley and sons, Inc., second edition,2005) 668-669.

[14]. ICH , International conference on harmonization, validation of analytical procedures; text and methodology Q2 (R1),2005 